\title{
纳米通道内混合气体流动的分子动力学模拟
}

\author{
解 辉 刘 朝* 刘涁武 \\ (重庆大学动力工程学院, 重庆 400030)
}

\begin{abstract}
摘要：采用非平衡分子动力学方法, 模拟了混合气体在纳米通道中的 Poiseuille 流动. 结果显示气体混合物 的化学成分与物理结构不再均匀一致, 随着亲水性气体粒子的减少, 亲水性粒子逐渐被吸附于壁面, 而疏水性 粒子主要分布于通道中间. 当亲水性粒子为 $10 \%$ 时, 混合气体在壁面处形成有序的“类固体”. 在本文的模拟条件 下, 流体速度分布显示混合气体流动速度随着疏水性粒子比例的增加而升高; 同时, 混合气体滑移速度也从负 滑移速度逐渐转变为正滑移速度.
\end{abstract}

关键词：纳米流动; 混合气体; 非平衡分子动力学; 纳米通道; Poiseuille 流动 中图分类号：0645；0641

\section{Molecular Dynamics Simulation of Gas Mixture Flow in Nanochannel}

\author{
XIE Hui LIU Chao * LIU Bin-Wu \\ (College of Power Engineering, Chongqing University, Chongqing 400030, P. R. China)
}

\begin{abstract}
Non-equilibrium molecular dynamic simulations have been carried out to study the Poiseuille flow of a mixture of gases in nanochannels. Results reveal that the chemical composition of these mixtures and their physical structures are not uniform. Hydrophilic particles were gradually absorbed by the channel wall as the proportion of hydrophilic particles decreased while the hydrophobic particles were mainly situated in the middle of the channel. At a proportion of $10 \%$ hydrophilic particles the gas mixture is solid-like adjacent to the wall. The distribution of velocity shows that the flow speeds up with an increase in hydrophobic particles and the slip velocity of gas mixtures changes gradually from negative to positive.
\end{abstract}

Key Words: Nano-flow; Gas mixture; Nonequilibrium molecular dynamics; Nanochannel; Poiseuille flow

MEMS/NEMS(微纳电子机械系统, micro/nano electro-mechanical system) 器件具有传统器件所不具 有的诸多优点 ${ }^{[1]}, 1959$ 年美国物理学家诺贝尔奖获 得者 Feynman 就提出了微型机械设想 ${ }^{[2]}$. 在 MEMS/ NEMS 纳米通道中, 气体的流动有着重要的意义, 这 些流动可能由压力、温度及密度梯度驱动, 著名的有 热流逸、扩散流动及 Poiseuille 流动(外力驱动下流 道中的不可压缩粘性流动)等. 国内外的学者对单一 成分的微流动已经做了大量工作 ${ }^{[3-7]}$, 相比之下, 对 于复杂的多种成分混合气体的微流动研究却鲜有
报道. 混合气体的微流动模拟需要增加边界条件以 及更多的参数描述流动状态, 这使得分析更加复 杂. 纳米尺度对流体性质的影响以及界面滑移现象 等都不同于宏观尺度, 常规的研究方法已经不能适 用. 这迫切需要我们从分子水平上分析混合气体在 纳米通道内的流动特性.

壁面分子与流体分子间的作用强弱对纳米通 道内流体的流动具有重要的影响 ${ }^{[8-10]}$. 通过活性杂质 可以控制流体与壁面的作用强度 ${ }^{[11]}$, 美国迈阿密大 学已经制造出对氢气/一氧化碳、氮气/氧气、氮气/

Received: November 26, 2008; Revised: December 30, 2008; Published on Web: February 11, 2009.

*Corresponding author. Email: liuchao@cqu.edu.cn; Tel: +8623-65112469.

国家自然科学基金(50376077, 50776101)资助项目

C) Editorial office of Acta Physico-Chimica Sinica 
甲烷等分别表现作用强弱差别较大的专利材料 ${ }^{[12]}$. 不同种类的流体分子与壁面分子的作用强弱不同, 作用较强时其液态表现为浸润性(亲水性), 较弱时则 为非浸润性(疏水性) ${ }^{[13]}$, 为了行文的简洁明了, 下文分 别简称为亲水性粒子和疏水性粒子. 本文采用非平 衡分子动力学方法, 模拟了壁面与气体混合物各成 分作用强弱不同时纳米通道中的 Poiseuille 流动.

\section{1 分子动力学模拟细节}

模拟的纳米通道内气体 Poiseuille 流动系统的 几何结构如图 1 所示. 图中白色原子为以铂为模型 的固体壁面, 两壁面都平行于 $x y$ 面, $x 、 y$ 方向为周期 性边界条件, $z$ 方向为固壁边界条件. 中间绿色原子 为疏水性粒子, 黄色原子为亲水性粒子, 构成混合气 体在质量力 $G$ (施加于每个粒子上的力)的驱动下流 动. 本文选取亲水粒子与疏水粒子不同比例的混合 气体为模拟对象在通道内形成 Poiseuille 流动.

理想溶液即每个成分满足 Lewis-Randall 法则 或Henry 定律的溶液, 理想溶液中不同种类分子的 相互作用和相同种类分子的相互作用相同 ${ }^{[14]}$. 本文 旨在研究混合气体成分与壁面作用强弱不同时的 流动特性, 而不是研究某具体混合物的流动, 故混 合气体成分采用与固壁不同作用强弱的氩原子模 型. 流体粒子之间作用采用截断及移位的 LennardJones $(\mathrm{LJ})$ 势能函数 ${ }^{[15]}$, 即:

$$
\begin{aligned}
& u\left(r_{i j}\right)= \\
& \begin{cases}4 \varepsilon\left[\left(\frac{\sigma}{r_{i j}}\right)^{12}-\left(\frac{\sigma}{r_{i j}}\right)^{6}-\left(\frac{\sigma}{r_{\mathrm{c}}}\right)^{12}+\left(\frac{\sigma}{r_{\mathrm{c}}}\right)^{6}\right], & r_{i j} \leq r_{\mathrm{c}} \\
0, & r_{i j}>r_{\mathrm{c}}\end{cases}
\end{aligned}
$$

其中 $u$ 为分子势能, $r$ 为分子间距, $\sigma$ 为尺寸参数, $\varepsilon$ 为势阱深度. 对于氩原子之间的作用, $\varepsilon=1.67 \times 10^{-21}$ $\mathrm{J}, \sigma=3.405 \times 10^{-10} \mathrm{~m}$. 为减少计算时间, 势能截断长 度取 $r_{\mathrm{c}}=3.0 \sigma$, 超过该距离分子间的相互作用力可 忽略不计.

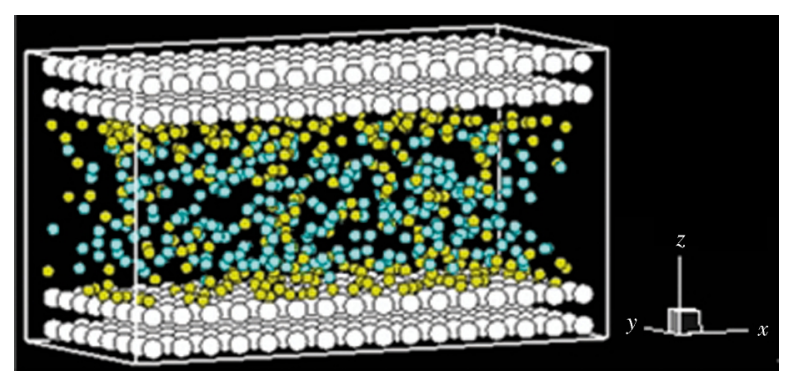

图 1 模拟系统的快照

Fig.1 A snapshot of the simulation system
为反映流体与通道壁面之间不同作用的强弱, 分子动力学模拟选取流体和固体壁面之间的 LJ 势 能函数为 ${ }^{[13]}$ :

$$
u_{\mathrm{ls}}\left(r_{i j}\right)=4 \alpha \varepsilon_{\mathrm{ls}}\left[\left(\frac{\sigma_{\mathrm{ls}}}{r_{i j}}\right)^{12}-\beta\left(\frac{\sigma_{\mathrm{ls}}}{r_{i j}}\right)^{6}\right]
$$

其中, $u_{\mathrm{ls}}$ 表示流体粒子和固壁粒子之间的 $\mathrm{LJ}$ 势能, $\varepsilon_{\mathrm{ls}}$ 和 $\sigma_{\mathrm{ls}}$ 表示流体粒子和固壁粒子之间的 LJ 作用 势能量和距离参数, 由 Lorentz-Berthelot 混合准则 确定 ${ }^{[16]}$.

$$
\varepsilon_{\mathrm{ls}}=\sqrt{\varepsilon_{1} \varepsilon_{\mathrm{s}}}, \sigma_{\mathrm{ls}}=\left(\sigma_{1}+\sigma_{\mathrm{s}}\right) / 2.0
$$

下标 1 、 $\mathrm{s}$ 分别表示流体、固壁, $\alpha$ 、 $\beta$ 用来调节流体粒 子和固壁粒子间的作用强弱, 以区分亲水性和疏水 性. 亲水性时取 $\alpha=1 、 \beta=1$, 疏水性时取 $\alpha=0.14 、 \beta=$ $0.1^{[13]}$.

系统在亲水粒子与疏水粒子不同比例的情况 下模拟，疏水粒子的百分比分别是 $25 \% 、 50 \%$ 、 $75 \% 、 90 \%$, 初始位置两种粒子均匀分布于流动空 间, 按 fcc 晶格排布. 采用简单速度标定方法 ${ }^{[17]}$, 将流 体和固体壁面的温度都控制为氩气模型的临界温 度 $T=150 \mathrm{~K}$. 流体粒子和固体粒子均为 2048 个, 固 壁粒子按 $\mathrm{fcc}$ 晶格排布, 流道在 $x 、 y$ 和 $z$ 方向尺度 为 $V=9.303 \times 4.652 \times 3.101 \mathrm{~nm}^{3}$. 对于粒子的动力学方 程, 采用 velocity Verlet 算法 ${ }^{[18]}$. 模拟初始阶段, 先让 系统达到给定热平衡态. 当系统大约运行一万步 时, 氩原子的速度分布符合 Maxwell-Boltzmann 速 度分布, 则可以认为系统达到平衡态. 随后每模拟 10 步施加质量力 $G=4.7374 \times 10^{-12} \mathrm{~N}$, 驱动系统偏离 平衡状态, 当系统再次达到稳态后, 统计参数计算 200000 步, 时间步长 $\Delta t=2.04 \times 10^{-14} \mathrm{~s}$.

在 $z$ 方向把纳米通道分为 20 层, 以便统计流体 在其中的密度、速度分布. 首先根据粒子在 $z$ 方向的 坐标统计每层的粒子个数 $N$. 然后根据式(4) ${ }^{[19]}$ 计算 每层在 $x$ 方向的流动速度 $V$ :

$$
V=\frac{1}{\left(J_{\mathrm{N}}-J_{\mathrm{M}}+1\right) \times N} \sum_{j=J_{\mathrm{M}}}^{J_{\mathrm{N}}} \sum_{i=1}^{N} v_{i, j}^{x}
$$

$v_{i, j}^{x}$ 表示粒子 $i$ 在 $j$ 步时 $x$ 方向的速度, $J_{\mathrm{M} 、} J_{\mathrm{N}}$ 分别表 示统计开始和结束的步数. 为了得到分子的运动轨 迹, 每 100 步记录一次粒子在 $x 、 y 、 z$ 方向的坐标位 置.

为了验证程序的正确性, 取亲水粒子为 $100 \%$ 时的情况, 设置与文献[20]中相同的参数运行程序, 取得的速度分布及密度分布与该文献中亲水性的模 拟结果相同, 由此证明了程序及统计方法的正确性. 

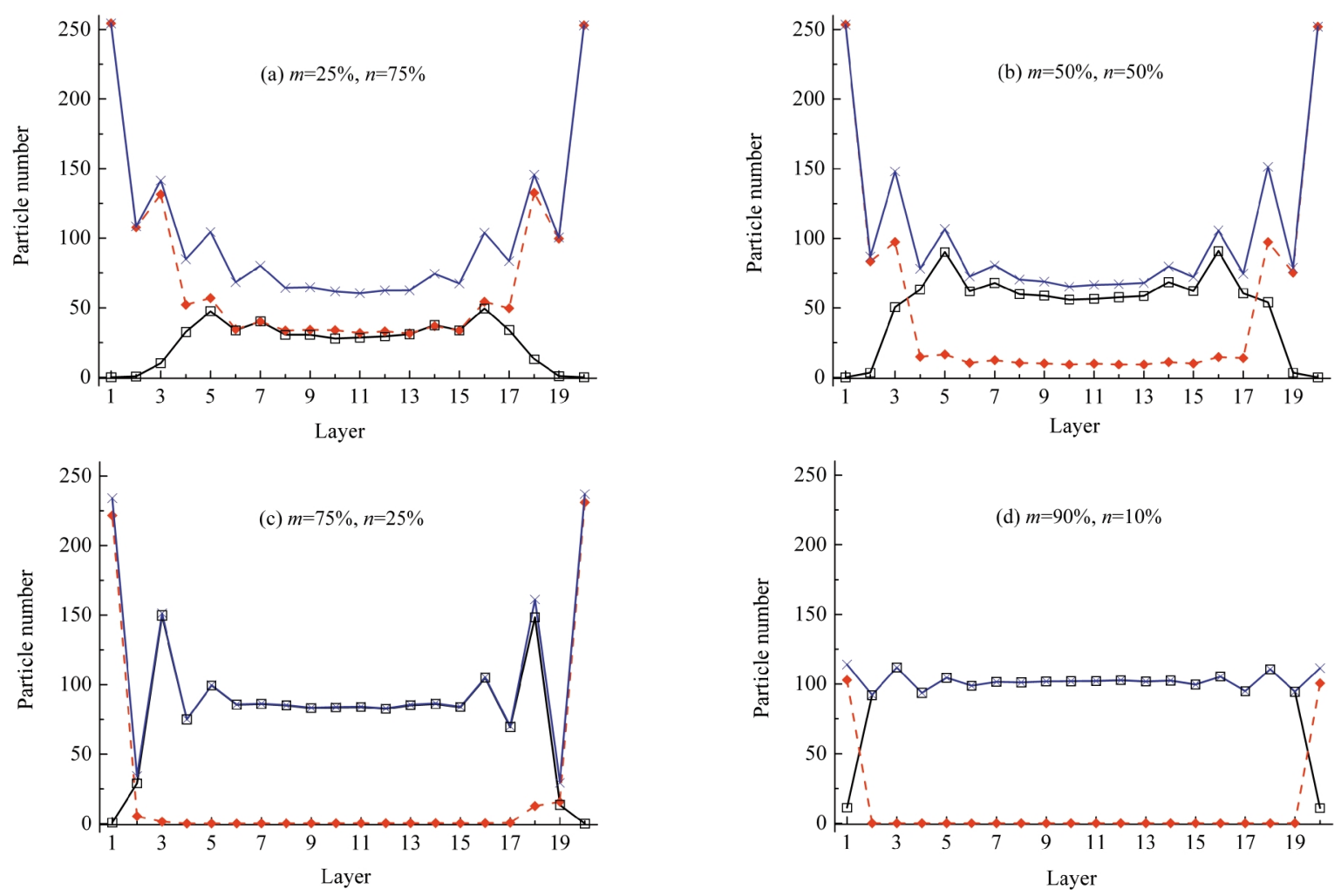

图 2 流体粒子分布图

Fig.2 Distribution of fluid particles

$m$ : the proportion of hydrophobic particles, $n$ : the proportion of hydrophilic particles; the volume of every layer is $(1 / 20) \times 9.303 \times 4.652 \times 3.101 \mathrm{~nm}$; $\square$ hydrophobic; $-\rightarrow$ hydrophilic; $\multimap$ total

\section{2 结果与讨论}

\section{1 流体粒子的分布}

亲水粒子与疏水粒子不同比例时, 流体粒子在 纳米通道内的密度分布如图 2 所示, 其中横坐标表 示 $z$ 方向的密度分层数, 纵坐标为数密度, 表示该 层内所对应的分子个数. 可以看出, 全部粒子的密 度分布在图 $2(\mathrm{a} 、 \mathrm{~b} 、 \mathrm{c})$ 中靠近壁面处较大, 而在图 2 (b、c)中靠近壁面的第二层较小, 为明显的分层现象, 在图 2(d)中壁面附近及管道中心的分布总体趋于 一致. 在图 2(a)和(b)中, 亲水性粒子的比例分别是 $75 \% 、 50 \%$, 即分别有 $1536 、 1024$ 个粒子, 此时亲水 粒子分布于纳米通道的全部空间, 疏水性粒子则主 要分布于中间区域, 在靠近壁面的两层内几乎没有 疏水性粒子. 而在图 2(c)和(d) 图中, 随着亲水粒子 数的减少, 由于亲水粒子与固壁粒子的作用较强, 逐 渐向壁面靠近. 在图 2(c), 亲水性粒子的比例为 $25 \%$, 即 512 个粒子, 几乎完全分布于靠近壁面的两层内. 在图 2(d)中, 亲水性粒子的比例为 $10 \%$, 即 204 个 粒子, 全部分布于靠近壁面的一层内. 在一般情况 下, 气体混合物化学成分和物理结构都是均匀一致
的, 可以认为是气相溶液 ${ }^{[14]}$. 然而在纳米通道中, 从 以上分析可知, 由于各种粒子与壁面的作用强弱不 同, 会有较明显的分离, 其热力学性质等不能再用 分析溶液的方法进行计算.

\section{2 分子运动轨迹}

比较分析各种情况下分子的运动轨迹发现, 当 亲水性粒子为 $10 \%$ 时, 亲水粒子的运动轨迹出现较 为特殊的现象, 如图 3(a)所示, 其运动轨迹围绕多 个平衡点振动, 振动情况接近晶体, 而平衡点的滑 动又接近液体. 其他情况下, 无论亲水粒子或疏水 粒子运动轨迹都近似于图 3(b), 显示流体分子轨迹 遍历整个空间. 根据广义物态理论 ${ }^{[21]}$, 一种状态对应 一定的序, 晶体和液体都有对应的衡量有序程度的 参数, 所以对于近壁面附近流体形成的有序态也被 称作“类固体” ${ }^{[22]}$. 曾有学者在研究聚合体在壁面附 近的作用时 ${ }^{[23,24]}$, 发现聚合体在壁面附近会出现从弱 相互作用向强相互作用的突跃. 本文中气体成分在 壁面附近形成“类固体”, 说明在亲水粒子、疏水粒 子以及壁面粒子的相互作用下, 在壁面附近发生了 弱相互作用向强相互作用的转变. 


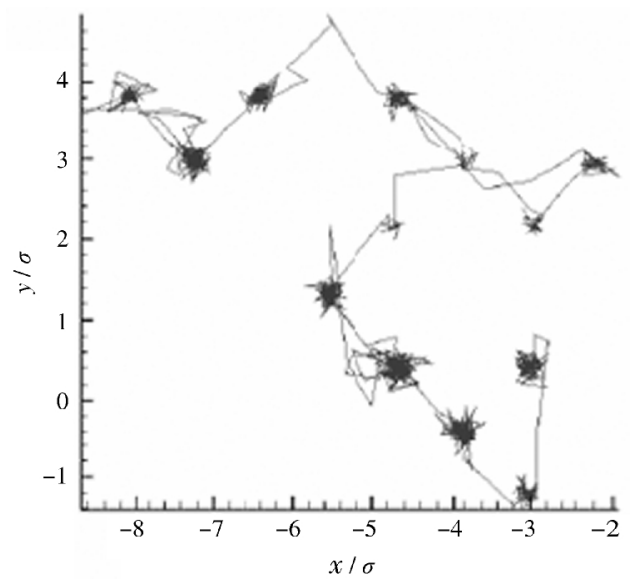

(a)

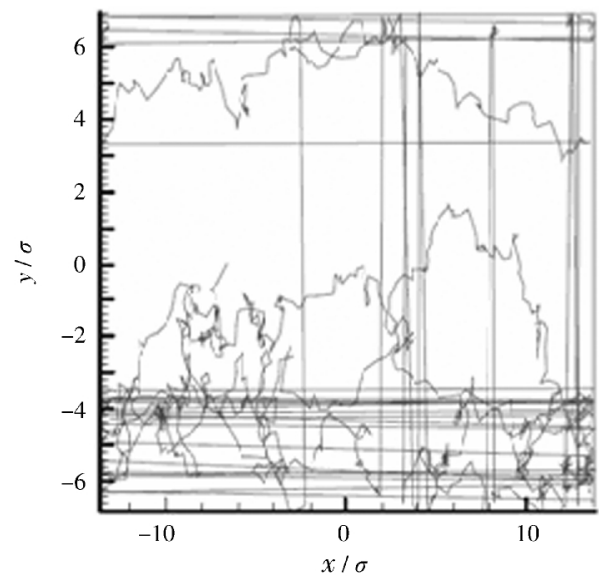

(b)

图 3 分子运动轨迹

Fig.3 Molecular trajectories

$\sigma$ : the molecular diameter of argon; (a) solid-like, (b) other particles

\section{3 速度与滑移}

与壁面作用强弱不同的混合气体, 在质量力 $G$ 的驱动下, 分子动力学模拟获得纳米通道内气体 Poiseuille 流动在 $x$ 方向的流动速度分布如图 4 所 示. 结果显示, 随着疏水性粒子比例的增加, “稀释”了 亲水性粒子与壁面的作用强度, 流动速度随之增加, 同时, 按照 Navier 边界条件的定义 ${ }^{[25,26]}$, 滑移速度为 固壁及其表面流体之间的速度差值, 可见滑移速度 也从负滑移速度逐渐转变为正滑移速度. 当出现无 滑移边界条件时, 速度分布满足 Navier-Stokes 理 论的速度分布. 仔细观察疏水性粒子比例为 $90 \%$ 及 $100 \%$ 时的速度分布, 可以发现前者的壁面附近第 一层粒子速度为零, 第二层即与后者第一层速度接 近, 通道中间的速度峰值也相同, 由上文分析知, 在

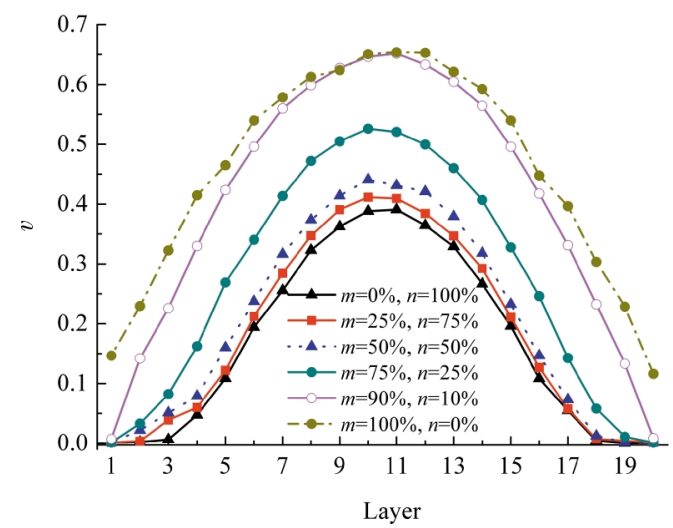

图 4 速度分布图

Fig.4 Velocity profiles

$m$ : the proportion of hydrophobic particles, $n$ : the proportion of hydrophilic particles, $v$ : the dimensionless velocity
疏水性粒子比例为 $90 \%$ 时, 亲水粒子在靠近壁面的 一层形成“类固体”, 可见疏水性粒子是在亲水性粒 子形成的类固体上滑移流动.

\section{3 结 论}

采用非平衡分子动力学方法, 模拟混合气体在 纳米通道中的 Poiseuille 流动. 得到了以下结论:

1) 在纳米通道中, 气体混合物的成分不再均匀 一致, 随着亲水性粒子的减少, 亲水性粒子逐渐被 吸附于壁面, 而疏水性粒子主要分布于通道中间.

2) 在亲水粒子、疏水粒子以及壁面粒子的相互 作用下, 混合气体在壁面附近发生了弱相互作用向 强相互作用的转变, 形成了“类固体”层.

3) 在本文的模拟条件下, 流体速度分布显示混 合气体流动速度随着疏水性粒子比例的增加而升 高, 同时, 滑移速度也从负滑移速度逐渐转变为正 滑移速度.

\section{References}

1 Li, D. S.; Guan, J. L.; Shi, Z. Y.; Zhang, J. H. Micro/nano technology and its application. Beijing: Science Press, 2005: 1-18 [李德胜, 关佳亮, 石照耀, 张建辉. 微纳米技术及其应用. 北京: 科学出版社, 2005: 1-18]

2 Feynman, R. P. J. Microelectromech. Syst., 1992, 1(1): 60

3 Panczyk, T. J. Comput. Chem., 2007, 28(3): 681

4 Soong, C. Y.; Yen, T. H.; Tzeng, P. Y. Phys. Rev. E, 2007, 76: 036303

5 Nedea, S. V.; Frijns, A. J. H.; van Steenhoven, A. A.; Jansen, A. P. 
J.; Markvoort, A. J.; Hilbers, P. A. J. J. Comput. Phys., 2006, 219

(2): 532

$6 \mathrm{Xu}, \mathrm{C}$; He, Y. L.; Wang, Y. Journal of Engineering Thermophysics, 2005, 26(06): 912 [徐超, 何雅玲, 王 勇. 工程热物理学报, 2005, 26(06): 912]

7 Cao, B. Y.; Chen, M.; Guo, Z. Y. Chin. Phys. Lett., 2004, 21(9): 1777

8 Markvoort, A. J.; Hilbers, P. A. J.; Nedea, S. V. Phys. Rev. E, 2005, 71: 066702

9 Nagayama, G.; Cheng, P. Int. J. Heat Mass Tran., 2004, 47(3): 501

10 Cao, B. Y.; Chen, M.; Guo, Z. Y. Journal of Engineering Thermophysics, 2003, 24(4): 670 [曹炳阳, 陈 民, 过增元. 工程热物理学报, 2003, 24(4): 670]

11 Karniadakis, G. E.; Beskok, A. Micro flows fundamentals and simulation. Trans. Institute of Process Engineering, Chinese Academy of Science. Beijing: Chemical Industry Press, 2006: 25-30 [微流动基础与模拟. 中国科学院过程工程研究所译. 北京: 化学工业出版社, 2006: 25-30]

12 Zhou, H.; Ma, S. Composition useful for selective gas adsorption comprises metal clusters; and amphiphilic ligands containing hydrophobic moiety, first hydrophilic moiety, and second hydrophilic moiety. U. S. Patent, US2008184881-Al. 2008

13 Cosgrove, J. A.; Buick, J. M.; Tonge, S. J.; Munro, C. G.; Greated, C. A.; Campbell, D. M. J. Phys. A-Math. Gen., 2003, 36(10): 2609

14 Su, C. S.; Tan, L. C.; Liu, G. Y. Higher engineering thermodynamics.
Beijing: Higher Education Press, 1987: 392-457 [苏长蒜, 谭连 城, 刘桂玉. 高等工程热力学. 北京: 高等教育出版社, 1987: 392-457]

15 Thompson, P. A.; Troian, S. M. Nature, 1997, 389(6649): 36

16 Allen, M. P.; Tildesley, D. J. Computer simulation of liquids. Oxford: Oxford University Press, 1989: 22-23

17 Sadus, R. J. Molecular simulation of fluids. Netherlands: Elsevier, 1999: 305-307

18 Swope, W. C.; Andersen, H. C.; Berens, P. H.; Wilson, K. R. J. Chem. Phys., 1982, 76(1): 637

19 Xu, J. L.; Zhou, Z. Q. Heat Mass Transfer., 2004, 40(11): 859

20 Liu, C.; Liu, B. W.; Zhang, H. L. Study of molecular dynamic simulation of Poiseuille flow in a microchannel. In: Proceedings of the micro/nanoscale heat transfer international conference 2008, Pts $\mathrm{A}$ and B. 1st ASME micro/nanoscale heat transfer international conference, Taiwan, 2008, New York: ASME, 2008: 649-654

21 Landau, L. D.; Lifshitz, E. M. Statistical physics. Trans. Yang, X. K. Beijing: People's Education Press, 1964: 224-269 [统计物理 学. 杨训恺译. 北京: 人民教育出版社, 1964: 224-269]

22 Granick, S. Science, 1991, 253(5026): 1374

23 Chakraborty, A. K.; Bratko, D. J. Chem. Phys., 1998, 108(4): 1676

24 Chakraborty, K.; Golumbfskie, A. J. Annu. Rev. Phys. Chem., 2001, 52: 537

25 Xu, J. L.; Li, Y. X. Int. J. Heat Mass Tran., 2007, 50(13-14): 2571

26 Cao, B. Y.; Chen, M.; Guo, Z. Y. Acta Phys. Sin., 2006, 55(10): 5305 [曹炳阳, 陈 民, 过增元. 物理学报, 2006, 55(10): 5305] 